\title{
A REALIDADE VIRTUAL IMERSIVA COMO TECNOLOGIA DE SUPORTE À COMPREENSÃO DE MODELOS COMPUTACIONAIS ${ }^{1}$
}

\author{
IMMERSIVE VIRTUAL REALITY AS A SUPPORT TECHNOLOGY TO THE \\ UNDERSTANDING OF COMPUTATIONAL MODELS
}

\author{
Daniel Carneiro Paes \\ Universidade Federal de Minas Gerais (UFMG) \\ danielcpaes@gmail.com \\ Eduardo Marques Arantes \\ Universidade Federal de Minas Gerais (UFMG) \\ arantes@demc.ufmg.br
}

\begin{abstract}
Resumo
Este artigo apresenta os resultados finais da pesquisa de mestrado que buscou verificar a capacidade de um sistema de Realidade Virtual Imersiva Exploratória em contribuir para uma melhor compreensão dos usuários sobre o modelo virtual. O método adotado define-se como qualitativo e exploratório e procurou comparar o nível de percepção entre duas situações: utilizando-se uma estação de trabalho convencional e o Ambiente de Imersão da Escola de Arquitetura e Design da UFMG (AIVITS). A coleta de dados baseou-se na submissão de Questionários de Verificação de Percepção. A partir da comparação entre as respostas coletadas foram identificadas as diferenças de percepção entre os modelos virtuais e suas relações com os diferentes perfis de participantes. O estudo concluiu que o Ambiente de Imersão favorece uma melhor percepção do espaço virtual em comparação com a percepção obtida utilizando-se o sistema não imersivo. Os usuários mais beneficiados pelo uso dessa tecnologia foram os homens, com idade igual ou superior a 26 anos, com $3^{\circ}$ grau ou pósgraduação, arquitetos ou engenheiros, sem daltonismo. Esses achados indicam que o Ambiente de Imersão é capaz de otimizar o processo de projeto, em especial, a atividade de análise crítica, conduzindo arquitetos e engenheiros à concepção de melhores soluções.
\end{abstract}

Palavras-chave: Realidade virtual. Ambientes de imersão. Processo de concepção. Percepção.

\begin{abstract}
This paper presents the final results of a master's research, which sought to verify the capacity of an Immersive Virtual Reality system in providing users with a better understanding of the virtual model. The adopted method is defined as qualitative and exploratory. It compares the level of perception between two situations: using a conventional workstation and the Immersive Environment of the School of Architecture of UFMG (AIVITS). Data collection was based on the submission of Perception Verification Questionnaires. From the comparison between the responses it was possible to identify differences of perception between the virtual models, as well as their relations to the different participants' profiles. The study concluded that the Immersive Environment promotes a better understanding of the virtual space compared to the perception obtained using the non-immersive system. The most benefited users from the use of this technology are the men aged over 26 years, with completed undergraduate or graduate degrees, architects or engineers, without color blindness. These findings indicate that the Immersive Environment can improve the design process, especially the activity of critical analysis, leading architects and engineers to the conception of better solutions.
\end{abstract}

Keywords: Virtual reality. Immersive environments. Design process. Perception.

${ }^{1}$ PAES, D.; ARANTES, E. A realidade virtual imersiva como tecnologia de suporte à compreensão de modelos computacionais. In: ENCONTRO BRASILEIRO DE TECNOLOGIA DE INFORMAÇÃO E COMUNICAÇÃO NA CONSTRUÇÃO, 7., 2015, Recife. Anais... Porto Alegre: ANTAC, 2015. 


\section{INTRODUÇÃO}

A evolução das tecnologias digitais de suporte ao processo de concepção de projetos evidencia uma tendência ao desenvolvimento de aplicações destinadas ao suporte à tomada de decisão. As decisões de maior repercussão nos custos, velocidade e qualidade dos empreendimentos são tomadas ainda na fase de concepção, onde a capacidade de influência sobre os custos totais da construção é maior (TOBIN, 2008; OWEN et al., 2010; MANZIONE et al., 2011).

Nesse contexto, o uso dos modelos computacionais tridimensionais contribui positivamente para uma melhor compreensão da configuração espacial e estrutural do ambiente representado e para o suporte ao processo decisório baseado em comparações visuais das soluções de projeto. Essa forma de expressão supera o desenho bidimensional principalmente devido ao seu aspecto interativo. No processo de concepção de projetos, a adoção de representações que se aproximam ao máximo da experiência existencialespacial é de fundamental importância para que sejam projetados espaços que de fato corresponderão técnica, funcional e simbolicamente ao uso destinado (MALARD; RHODES; ROBERTS, 1997).

A escassez de estudos sobre aplicações de realidade virtual imersiva aos processos da indústria da AEC evidencia a necessidade de mais investigações. Nessa direção, o objetivo geral dessa pesquisa é avaliar a capacidade do Ambiente de Imersão Virtual de Tecnologia Simplificada (AIVITS), um sistema de Realidade Virtual Imersiva Exploratória, em contribuir para uma melhor percepção do espaço virtual, em comparação à percepção obtida através da realidade virtual não imersiva (estação de trabalho convencional).

A compreensão/percepção do modelo virtual é necessária ao longo de todo o processo de concepção do projeto do edifício, mais especificamente nos momentos de realização da análise crítica distribuídos ao longo da projetação, quando os projetistas submetem seus projetos à própria avaliação e à de terceiros, através de representações computacionais. $\mathrm{A}$ análise crítica consiste numa avaliação sistematizada e documentada quanto à pertinência, adequação e eficácia das soluções de projeto em atender aos seus requisitos. Procura identificar possíveis falhas no projeto propondo soluções para corrigi-las (ROMANO, 2006; SILVA; NOVAES, 2008).

O método de pesquisa adotado neste trabalho define-se como qualitativo e exploratório. Procura realizar uma comparação do nível de percepção sobre o objeto representado virtualmente (ambiente interior de um edifício), entre duas situações: utilizando-se infraestrutura para Realidade Virtual não Imersiva (RVnl) e para Realidade Virtual Imersiva (RVI). A aplicação dos Questionários de Verificação de Percepção (QVP) permitiu avaliar o grau de percepção dos participantes. A resposta a uma determinada questão do QVP aplicado em um dos ambientes virtuais (RV não Imersiva ou RVI) foi comparada com a resposta à mesma questão quando aplicada na visita ao ambiente físico. A compatibilidade entre as respostas das duas situações (ambiente virtual e ambiente físico) indica a capacidade daquela tecnologia em reproduzir a percepção obtida no ambiente físico. A partir da comparação dessa compatibilidade entre as duas tecnologias, foi possível se verificar qual delas oferece uma percepção do espaço virtual mais próxima da percepção do espaço físico (OLIVEIRA, 2003; FRACAROLI, 2006).

\section{FUNDAMENTAÇÃO}

\subsection{0 processo de concepção}

O processo de concepção implica em um processamento mental de informações altamente complexo e sofisticado. Envolve etapas de percepção, identificação, interpretação, reflexão 
e codificação, utilizando-se dos sentidos da emoção, da razão e da linguagem. Para se descrever um problema mal definido com detalhes suficientes para sua compreensão, é preciso se desenvolver um exaustivo inventário sobre todas as suas possíveis soluções. $O$ problema vai se definindo com o aumento de suas especificações formais, enquanto ele é gradativamente resolvido (CHANDRASEGARAN et al., 2013).

Malard (2004) realiza uma comparação entre a pesquisa científica e a "invenção arquitetônica". Segundo a autora, "o processo de projeto é um processo de aquisição ou de produção de conhecimento sobre o objeto que se projeta". Para Florio (2011), tanto no processo de concepção arquitetônica quanto no pensamento científico é preciso operar para além dos limites do conhecimento atual, pela proposição de hipóteses, de métodos investigativos e de experimentação. Projetistas pensam o que fazem, enquanto o fazem. Não há um planejamento prévio para aquilo que há de se conceber, mas expectativas e objetivos. A pesquisa em arquitetura seria um processo de reflexão-na-ação (SCHÖN, 2000).

No processo de projeto um primeiro sujeito parte para o conhecimento de um objeto abstrato que lhe ocupa a mente. Ele assimila esse objeto descrevendo e representando-o, seja em sua mente ou sob a forma de um modelo virtual, por exemplo (ALVARENGA, 2003). Um modelo pode oferecer suporte à comunicação, aprendizagem e à análise sobre aspectos relevantes do contexto que ele procura representar; pode servir como um veículo para o raciocínio, para a resolução de problemas e para aquisição de novos conhecimentos (GUIZZARDI, 2005). A representação bidimensional não é capaz de conter toda a complexidade da arquitetura como também as possibilidades de soluções (ISAACS et al., 2011). Por outro lado, a exploração de modelos tridimensionais interativos permite a compreensão das complexas questões do projeto (BRADY, 1997).

De acordo com Chandrasegaran et al. (2013), as ferramentas destinadas ao projeto conceitual são mais recentes e surgiram a partir de 1995. Por sua vez, as ferramentas de croquis digitais são ainda mais recentes, o que demonstra um reconhecimento da importância dessa forma de conhecimento desestruturado no processo de projeto. Muitas ferramentas de CAD vêm sendo continuamente modificadas a fim de incorporar recursos para o desenvolvimento de projetos conceituais.

\subsection{A Realidade Virtual}

Bertol (1997) define realidade virtual como um mundo gerado por computador, onde é possível a experiência imersiva em tempo real. À sensação de imersão no ambiente, a autora atribui a expressão "senso de presença", fator determinante para a criação de uma realidade virtual separada do mundo "real". Para Faas et al. (2014), os elementos-chave da realidade virtual são o mundo/lugar virtual, o senso de presença, a resposta sensorial e a interatividade. Ainda segundo os autores, diferentes fatores são determinantes para se experimentar o senso de presença, tais como: noção espacial, qualidade de imersão, envolvimento, qualidade da interface, navegabilidade no ambiente virtual, veracidade, interatividade e previsibilidade.

Steuer (1992) define realidade virtual como um tipo particular de experiência humana partindo do conceito de "presença" (presence). Para o autor, presença é a sensação de estar presente em um ambiente a partir da percepção do mesmo, mediada por processos mentais. Quando a percepção é auxiliada por uma tecnologia de comunicação, diz-se "telepresença". Telepresença é a experiência de presença em um ambiente através de um meio de comunicação. Por conseguinte, realidade virtual seria "toda e qualquer experiência de telepresença". Tal definição transfere o termo realidade virtual da ideia de um conjunto específico de hardware para a ideia de percepções de um indivíduo (STEUER, 1992). 


\subsection{A Realidade Virtual Imersiva e os Ambientes de Imersão}

A natureza da experiência humana em ambientes virtuais se relaciona com a definição de presença do indivíduo nesses espaços. Quando um modelo tridimensional é apresentado em um monitor convencional a visão binocular humana - capaz de identificar profundidades - informa que o objeto visualizado se trata de uma imagem plana. A noção de profundidade ocorre quando no espaço tridimensional os objetos aparecem e desaparecem atrás uns dos outros ao movimento do observador. Se um modelo tridimensional é apresentado em formato estereoscópico por uma grande tela panorâmica, cria-se a ilusão de superfícies em profundidade. Perceber a si mesmo inserido no ambiente é a raiz do senso de presença em Ambientes de Imersão (WANN; MON-WILLIAMS, 1996).

A diferença mais evidente entre as formas imersiva e não imersiva da realidade virtual é exatamente o grau de imersão oferecido pelo funcionamento concomitante de software e hardware em cada uma das plataformas. O grau de imersão pode ser determinado pela intensidade da sensação de imersão ou do senso de presença em um ambiente de realidade virtual. Nesse contexto, pelo uso de aplicativos e equipamentos sofisticados, as plataformas de realidade virtual imersiva (Ambientes de Imersão ou, do inglês, Immersive Environments) são capazes de oferecer ao usuário simulações mais fiéis à sua experiência no mundo físico, mais intensas em termos do senso de presença. Altos níveis de presença em ambientes virtuais imersivos indicam que o projetista está altamente focado em seu trabalho e têm relação direta com a alta performance dos projetos e com as melhores soluções. Em suma, a realidade virtual, por definição, considera o senso de presença, ao passo que a realidade virtual imersiva procura intensificar esta sensação, incrementando a experiência espaço-temporal do usuário (BERTOL, 1997; FAAS et al., 2014; MALARD; RHODES; ROBERTS, 1997).

A profunda percepção do projeto oferecida pelo Ambiente de Imersão é capaz de comunicar informações relevantes de forma mais eficiente e com menor deturpação de conteúdo em comparação às técnicas tradicionais de visualização tridimensional (KALISPERIS et al., 2002). O senso de presença que surge pela utilização de ambientes de imersão transforma a maneira como os dados são explorados, contribuindo para o processo mental de assimilação de informações complexas. Usuários são capazes de ver, navegar e interagir com os dados de uma forma intuitiva, em um contexto completamente tridimensional que preserva relações espaciais fundamentais para o reconhecimento do objeto. $O$ uso desses ambientes virtuais colaborativos (do inglês, Collaborative Virtual Environments - CVE) permite que usuários em diferentes localizações físicas interajam entre si e com os dados compartilhados em um mundo virtual (LASCARA et al., 1999).

Sistemas imersivos de realidade virtual baseados em projeções (projection-based systems), como a CAVE (Cave Automatic Virtual Environment), permitem que o usuário participe diretamente na cena gerada por computador, através de uma experiência completamente imersiva, com exploração do ambiente pelo movimento da cabeça, navegação pelo caminhar e manipulação dos objetos virtuais por gestos das mãos e dedos (CRUZ-NEIRA, 1996). Multinacionais como Boeing, Volkswagen, Chrysler, Ford, Caterpillar e GM estão adotando as CAVEs em seus processos produtivos com o objetivo de reduzir o número de protótipos físicos para avaliação de designs intermediários. Ao adotar essa tecnologias, as empresas podem utilizar diversos protótipos virtuais para avaliação e simulação do produto em desenvolvimento, economizando tempo e dinheiro e contribuindo para melhores produtos finais, tonando-se mais competitivas (RYKEN; VANCE, 2000).

Entretanto, as CAVEs ainda são sistemas de custo muito elevado e portanto, restritos a poucos grupos de pesquisa ou segmentos industriais. Poucos anos após o surgimento da primeira CAVE, ambientes de imersão de baixo custo ou de tecnologia simplificada começaram a ser desenvolvidos. Esses sistemas empregam tecnologias e componentes de 
hardware de menor custo e já disponíveis no mercado e exigem habilidades computacionais menos avançadas para serem operados, tornando-se acessíveis a uma variedade maior de usuários (KALISPERIS et al., 2002).

\section{MÉTODO}

A tecnologia utilizada na pesquisa para o caso do ambiente de Realidade Virtual não Imersiva é constituída de uma estação de trabalho convencional composta por um computador de alto desempenho, monitor LCD, teclado e mouse. Para o caso do ambiente de Realidade Virtual Imersiva foi utilizado o AIVITS, Ambiente de Imersão Virtual de Tecnologia Simplificada da Escola de Arquitetura e Design da UFMG, composto pelo mesmo computador de alto desempenho citado anteriormente, três módulos de projeção idênticos equipados com um projetor estereoscópico e um espelho cada, uma grande tela panorâmica de tecido sintético com dimensões de $1,70 \mathrm{~m}$ de altura por $6,81 \mathrm{~m}$ de largura, óculos de estereoscopia passiva (filtro simples), teclado e mouse. O objetivo geral do AIVITS é viabilizar a experiência virtual imersiva no âmbito da representação tridimensional e da interpretação de projetos de construção civil. Os aspectos técnicos referentes aos softwares, hardwares e demais equipamentos do AIVITS se basearam nas configurações do projeto VR-Desktop, da Universidade Estadual da Pensilvânia (Penn State University), EUA.

O esquema de funcionamento do sistema é apresentado na Figura 1. A maquete virtual é desenvolvida no software Sketch Up e exportada para o Unity, capaz de criar o par de imagens estereoscópicas e uni-las em um anáglifo (modelo estereoscópico). Este procedimento é realizado utilizando-se o computador mencionado anteriormente que também realiza a distribuição dessa imagem para os três projetores estereoscópicos, a partir de suas três placas de vídeo. A imagem é então projetada simultaneamente sobre os espelhos dos módulos de projeção e rebatida para a tela panorâmica. Utilizando-se dos dispositivos de navegação (teclado e mouse convencionais) e dos óculos de filtro simples o usuário é capaz de se locomover através do espaço virtual percebendo profundidades.

Figura 1 - Esquema de funcionamento do AIVITS

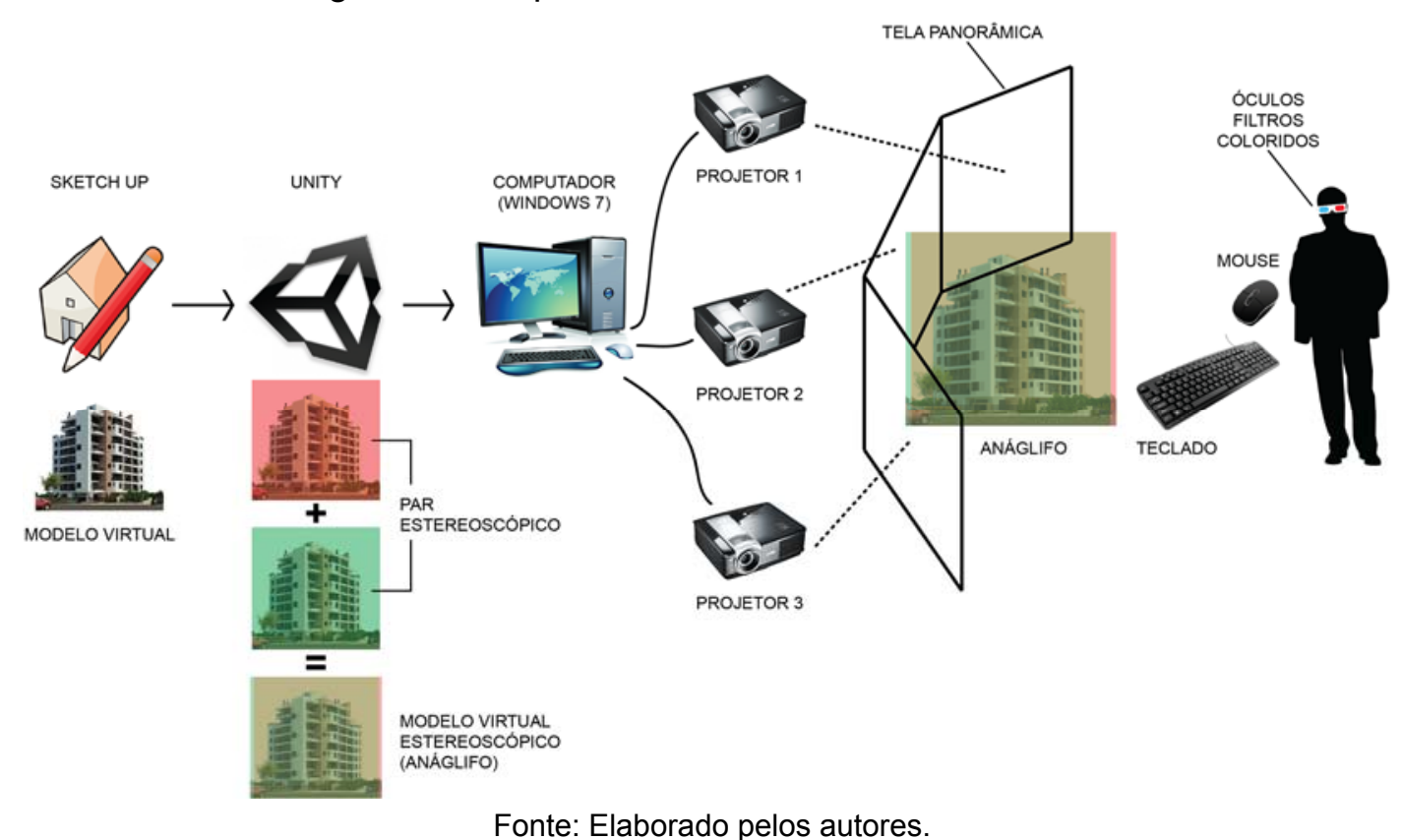

O procedimento de coleta de dados para este trabalho se baseou na submissão dos Questionários de Verificação de Percepção (QVP) como os desenvolvidos por Witmer e 
Singer (1998), Oliveira (2003), Fracaroli (2006) e Faas et al. (2014). Outro questionário utilizado no experimento, intitulado Questionário de Caracterização do Perfil do Participante (QCPP), procurou coletar características individuais que pudessem funcionar como filtros condicionantes do nível de percepção do espaço pelo usuário.

O procedimento experimental completo foi realizado nas seguintes etapas: 1) Definição do ambiente de estudo; 2) Desenvolvimento das maquetes/ambientes virtuais; 3) Elaboração dos questionários (QCPP e QVPs); 4) Ensaio do experimento; 5) Definição e caracterização dos participantes, utilizando-se o QCPP; 6) Apresentação da animação a cada um dos participantes em RV não Imersiva e aplicação concomitante do QVP RVnl; 7) Apresentação da animação a cada um dos participantes em RVI e aplicação concomitante do QVP RVI; 8) Visita do participante ao ambiente físico e aplicação concomitante do QVP AF; 9) Análise dos dados coletados.

$\mathrm{Na}$ etapa 2 foram confeccionadas as maquetes virtuais para cada plataforma, RV não Imersiva e RVI. Primeiramente, a partir de desenhos técnicos do projeto de reforma do edifício, foi elaborada uma maquete virtual do ambiente utilizando-se o software Sketch Up Pro (versão 8.0.3117), para os testes com RV não Imersiva. Esta maquete foi então exportada em extensão .3ds e importada pelo Unity (versão 4.0.1F2). Neste software foi possível criar uma maquete em formato estereoscópico (o anáglifo) que serviria posteriormente para os testes com RVI.

Optou-se neste trabalho pelo desenvolvimento de animações do tipo exploratória e simplificada - aquela em que o usuário pode explorar o ambiente virtual definindo seu próprio percurso, com grau de realismo baixo, sem recursos de rendering como luz, sombra e texturas em relevo. Tem-se portanto a criação de dois ambientes de realidade virtual:

1 Ambiente de Realidade Virtual não Imersiva exploratória simplificada (RVnl - estação de trabalho convencional);

2 Ambiente de Realidade Virtual Imersiva exploratória simplificada (RVI - AIVITS).

Na etapa 3 foram elaborados os questionários de Caracterização do Perfil do Participante (QCPP) e de Verificação de Percepção (QVP). Os QVPs são compostos por perguntas objetivas que procuraram coletar a noção espacial dos participantes, num total de sete questões que se repetem em cada um dos três QVPs (RVnl, RVI e AF). As questões procuraram estimular a percepção do participante em relação a: distâncias verticais, formato e área do espaço (pela avaliação da capacidade de lotação), quantidade e localização espacial de elementos, distâncias horizontais e distância entre elementos. Evitou-se a inclusão de questões subjetivas aos QVPs após a constatação na bibliografia de que este tipo de questão poderia conduzir a dados de baixa relevância ou a conclusões inconsistentes.

Para cada ambiente visitado foi desenvolvido um QVP, sendo portanto, três questionários diferentes: o QVP RVnl destinado aos testes no ambiente de Realidade Virtual não Imersiva, o QVP RVI para o ambiente de Realidade Virtual Imersiva e o QVP AF, utilizado no Ambiente Físico. Os QVPs utilizados nos testes dos ambientes de realidade virtual (QVP RVnl e QVP RVI) possuíam sete questões idênticas, mas ordenadas de forma diferente. $O$ QVP utilizado nos testes do ambiente físico (QVP AF) possuía as mesmas sete questões ordenadas também de outra forma - além de outras duas questões adicionais, totalizando nove questões. Para exemplificar o caráter e a estrutura das questões e das alternativas de respostas dos QVPs, uma delas é reproduzida a seguir: "A distância máxima entre o piso e o teto do ambiente é de: a) até 4 metros; b) até 6 metros; c) até 8 metros; d) até 10 metros; e) até 12 metros; f) não consigo avaliar".

As etapas 5, 6, 7 e 8 se referem aos procedimentos de coleta de dados e foram realizadas nesta ordem, com cada um dos participantes. O participante foi apresentado às duas 
animações - primeiro à animação não imersiva, depois à animação imersiva. O participante explorava cada um dos ambientes virtuais até que respondesse a todas as questões do QVP (RVnl ou RVI). Da mesma forma, cada participante visitava o ambiente físico e respondia ao QVP AF. A Figura 2 apresenta momentos do procedimento de coleta de dados enquanto um participante explorava a animação em realidade virtual não imersiva (à esquerda) e em seguida a animação em realidade virtual imersiva (à direita) com o uso dos óculos de estereoscopia passiva.

Figura 2 - Participante navegando pela animação em RVnl (esq.) e em RVI (dir.)

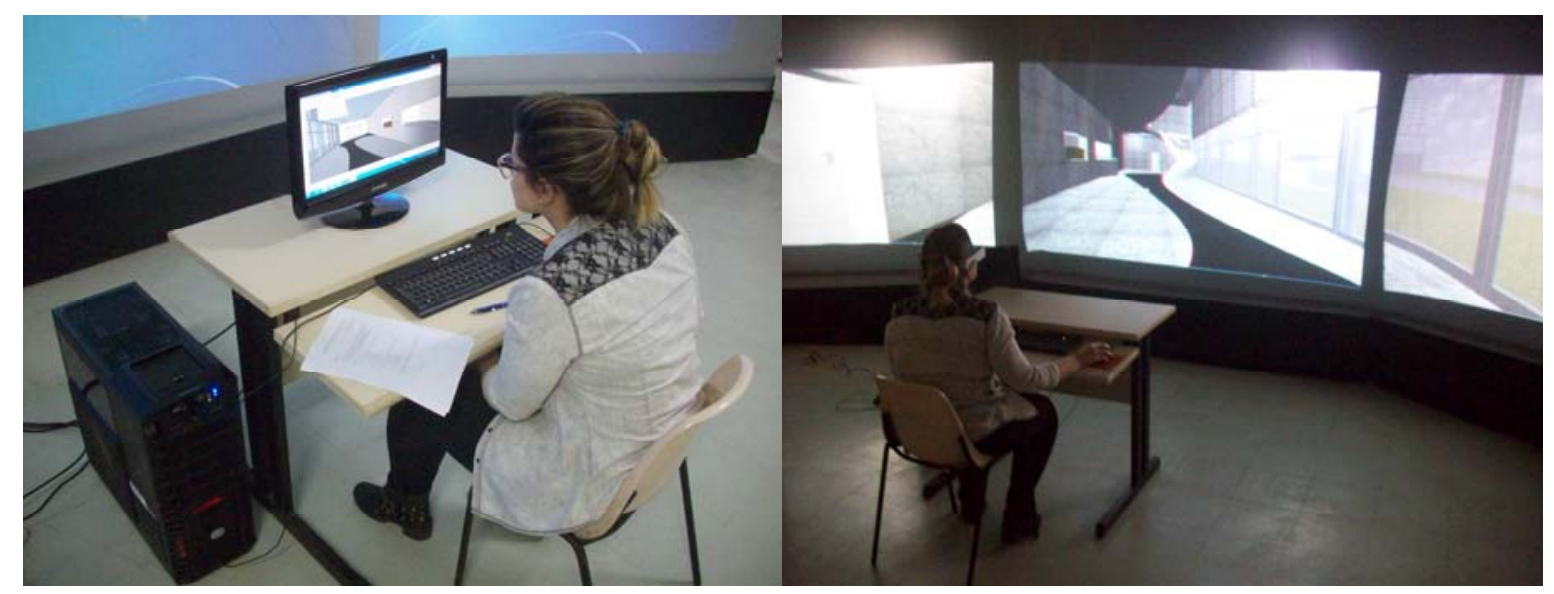

Fonte: Elaborado pelos autores.

$\mathrm{Na}$ etapa 9 foram realizadas as comparações entre as respostas obtidas sobre a percepção dos ambientes virtuais com as respostas obtidas sobre a percepção do ambiente físico. Essas comparações foram realizadas com o objetivo de se obter os Índices de Manutenção da Percepção (IMP) que serviriam para todas as análises posteriores. A resposta a uma determinada questão do QVP aplicado em um dos ambientes virtuais (RV não Imersiva ou $\mathrm{RVI}$ ) foi comparada com a resposta à mesma questão quando aplicada na visita ao ambiente físico. Para essas comparações, foi estabelecida uma escala de valores numéricos para cada uma das possíveis respostas de cada questão, apresentada na Tabela 1 (FRACAROLI, 2006). A compatibilidade entre as respostas das duas situações indica a capacidade daquela tecnologia em reproduzir a percepção obtida no ambiente físico, considerando-se que essa capacidade existe quando as respostas são idênticas ou não existe quando elas forem diferentes.

Tabela 1 - Escala de valores numéricos das respostas das questões do QVP

\begin{tabular}{|c|c|}
\hline Resposta & Valor numérico \\
\hline a) & 1 \\
\hline b) & 2 \\
\hline c) & 3 \\
\hline d) & 4 \\
\hline e) & 5 \\
\hline f) & sem valor \\
\hline
\end{tabular}

Fonte: Elaborado pelos autores.

Subtraindo-se o valor numérico da resposta à uma determinada questão aplicada na visita ao ambiente virtual do valor numérico da resposta à mesma questão aplicada na visita ao ambiente físico, obtém-se o nível de semelhança entre as percepções nas diferentes situações, como apresentado na Tabela 2, que é o Índice de Manutenção da Percepção (IMP) citado anteriormente (OLIVEIRA, 2003). 


\section{霞}

Tabela 2 - Exemplo de cálculo do Índice de Manutenção da Percepção

\begin{tabular}{|c|c|c|c|c|}
\hline \multicolumn{5}{|c|}{ Participante $n^{\circ} X$} \\
\hline \multirow{2}{*}{ Questão do QVP } & \multicolumn{2}{|c|}{ Valor numérico da resposta escolhida } & \multirow{2}{*}{$\begin{array}{l}\text { Diferença } \\
\text { (AV) - (AF) }\end{array}$} & \multirow{2}{*}{ IMP } \\
\hline & Ambiente Virtual (AV) & Ambiente Físico (AF) & & \\
\hline 1 & 2 & 5 & $2-5=-3$ & 3 \\
\hline 2 & sem valor & 3 & sem valor & sem valor \\
\hline 3 & 3 & 1 & $3-1=2$ & 2 \\
\hline 4 & 5 & 5 & $5-5=0$ & 0 \\
\hline 5 & 1 & 2 & $1-2=-1$ & 1 \\
\hline
\end{tabular}

Fonte: Elaborado pelos autores.

Quanto mais próximo de zero for o valor absoluto dessa operação, maior é o nível de semelhança entre as percepções. Neste trabalho são analisados apenas os IMPs nulos (diferença igual a zero), pois são representantes de uma percepção idêntica entre as duas situações (ambiente físico versus ambiente virtual). Por exemplo: se o valor da resposta à questão 1 na visita ao ambiente virtual for 2 e o valor da resposta à mesma questão na visita ao ambiente físico também for 2 (mesmas respostas), o resultado da subtração desses valores será nulo, ou seja, o Índice de Manutenção da Percepção é igual a zero e a percepção sobre aquela questão nas duas situações será idêntica. Considera-se que a tecnologia de realidade virtual que obtiver um número maior de resultados nulos é aquela que oferece a percepção sobre o ambiente virtual mais semelhante à percepção sobre o mesmo ambiente em seu formato físico. Em outras palavras, é a tecnologia que oferece a experiência mais próxima da experiência "real".

\section{RESULTADOS E DISCUSSÃO}

Esta pesquisa contou com a participação de 30 pessoas, entre profissionais da construção civil (arquitetos e engenheiros), engenheiros de outras áreas envolvidos com atividades de projeto, estudantes de arquitetura e leigos (de outras profissões). A média de idade da amostra é de 28 anos. A maioria dos participantes se localiza entre as idades de 25 e 31 anos (70\% da amostra). $43 \%$ dos participantes são mulheres e os $57 \%$ restantes são homens. Participantes com pós-graduação representam $40 \%$ da amostra, com $3^{\circ}$ grau são $37 \%$ e com $2^{\circ}$ grau, $23 \%$. Arquitetos ou engenheiros compõem $44 \%$ da amostra, estudantes de arquitetura são $23 \%$ e profissionais de outras profissões, considerados "leigos" nesta pesquisa, são os restantes $33 \%$. Apenas $7 \%$ dos participantes declararam-se daltônicos. 0 percentual de participantes com familiaridade com recursos e ferramentas computacionais de representação tridimensional é de $60 \%$ e com conhecimento prévio do ambiente estudado (ambiente físico) foram $63 \%$.

Neste artigo apresenta-se apenas o principal resultado das análises, referente à Manutenção da Percepção Global (MPG), determinada em termos da relação entre IMPs nulos e não nulos. Se o índice é nulo, manteve-se a percepção, caso contrário não se manteve. A diferença das porcentagens de IMPs nulos entre as duas tecnologias (RVnl e $\mathrm{RVI}$ ) representa a MPG. Dentre todos os Índices de Manutenção da Percepção analisados para a relação RVnl x AF (209 IMPs), 60\% foram iguais a zero (nulos), sendo $40 \%$ diferentes de zero (não nulos). Para a relação RVI x AF, $71 \%$ foram nulos e $29 \%$ não nulos. A diferença das porcentagens de IMPs nulos entre as duas tecnologias (RVI e RVnl) é de $11 \%(71 \%-60 \%=11 \%)$, como mostra a Figura 3 a seguir. 
Figura 3 - IMPs nulos e não nulos entre RVnl e RVI

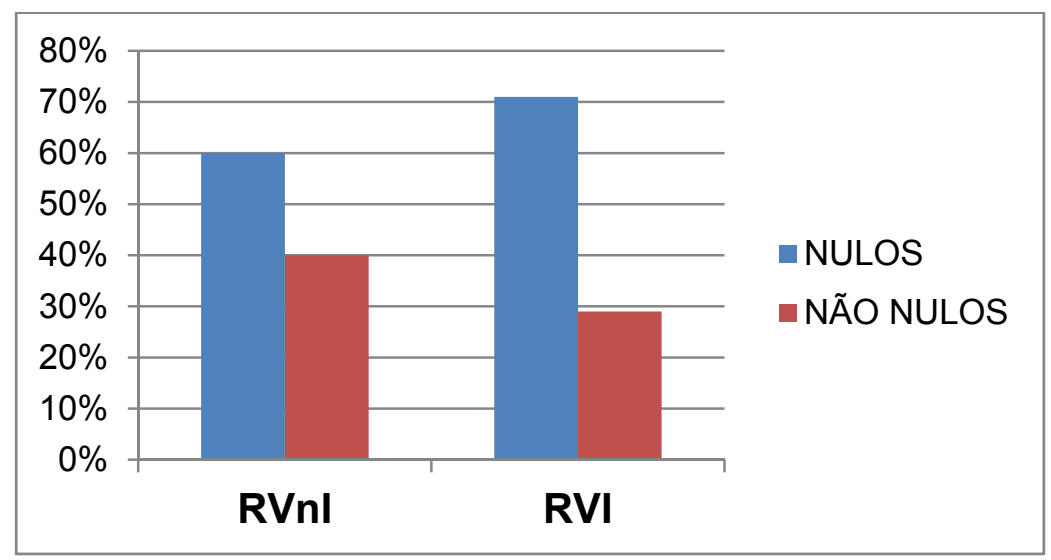

Fonte: Elaborado pelos autores.

Para determinação da significância dessa diferença, foi utilizado o software de estatística Estatcamp Action 2.8 (software livre). Realizou-se o Teste de Proporção Entre Duas Amostras, capaz de testar uma hipótese comparando as proporções de duas populações independentes. Realizando-se o teste entre as duas populações de IMPs nulos e não nulos (60\% / 40\% para RVnl e $71 \%$ / 29\% para RVI) obteve-se um p.valor igual a 0,018 . O p.valor representa a probabilidade da diferença observada entre as tecnologias ser devida ao acaso. Essa probabilidade de $1,8 \%$ é menor que o nível de significância limite adotado no teste, de 0,05 ou $5 \%$. Assim, pode-se afirmar com $95 \%$ de confiança que a diferença de $11 \%$ não foi devida ao acaso, ou seja, ela é significativa.

Uma análise mais detalhada sobre a Manutenção da Percepção foi realizada definindo-se a grupos de participantes segundo os filtros de percepção de Okamoto (1999) - faixa etária, gênero, escolaridade, daltonismo, familiaridade com recursos 3D, conhecimento prévio do ambiente estudado (ambiente físico) - acrescidos do filtro "profissão" e utilizando-se o mesmo procedimento estatístico para determinação da significância do que foi observado. A Manutenção da Percepção por Filtro Condicionante permitiu investigar a relação desses filtros com a frequência de IMPs nulos (manutenção da percepção) tanto em uma determinada tecnologia como também entre ambas. Assim, foi possível traçar um perfil de usuário para o qual o Ambiente de Imersão estudado é especialmente benéfico. Este usuário deve ter 26 anos ou mais, do sexo masculino, com $3^{\circ}$ grau ou pós-graduação, arquiteto ou engenheiro e não possuir daltonismo. Ter o conhecimento prévio do ambiente ou familiaridade com recursos e ferramentas de modelagem tridimensional não é critério para que o usuário descrito possa ter sua percepção melhorada ao utilizar o sistema imersivo.

\section{CONCLUSÕES}

As diferenças significativas de IMPs nulos entre as duas tecnologias levam à conclusão de que utilizando-se o Ambiente de Imersão obtém-se uma melhor percepção do ambiente virtual em comparação com a percepção obtida com o uso de uma estação de trabalho convencional. É importante ressaltar que essa melhora da percepção acontece com maior ênfase para o grupo profissional de arquitetos e engenheiros, envolvidos com a atividade de projeto.

Neste trabalho, verificou-se o senso de presença no Ambiente de Imersão em termos da percepção espacial do usuário. Os resultados acusaram uma melhor noção espacial com o uso do Ambiente de Imersão, portanto, um nível de presença mais elevado. O nível de presença tornou possível a identificação de determinados aspectos do espaço de forma 
mais precisa em comparação com a identificação obtida com o uso do sistema tradicional. Essa maior precisão implica em uma melhor compreensão do projeto, para além do simples reconhecimento da forma tridimensional em direção à assimilação de informações complexas e multidimensionais que surgem das relações entre os elementos distribuídos no espaço.

Como discutido por Faas et al. (2014), altos níveis de presença estão relacionados a um forte engajamento do projetista durante a atividade de projeto e têm relação direta com as melhores soluções. Assim, o nível de presença pode servir como um indicador de performance em atividades de projeto: se o projetista está "muito engajado" ou "presente" na atividade, melhores soluções surgirão. Na medida em que oferece maior nível de presença, o Ambiente de Imersão torna-se uma ferramenta potencialmente capaz de melhorar a performance dos projetistas, torná-los mais envolvidos com a atividade, levando-os à proposição de melhores soluções.

O Ambiente de Imersão representa a tecnologia mais avançada e de maior contribuição à etapa do projeto conceitual, à geração de ideias, onde acontecem as decisões de maior repercussão na qualidade do empreendimento. Uma melhor noção espacial, oferecida pela tecnologia, implica em uma melhor compreensão da representação, do espaço virtual. No Ambiente de Imersão, é possível se determinar a pertinência e adequação das soluções representadas a partir da experiência existencial-espacial, para além da simples comparação visual. $\mathrm{Na}$ medida em que o problema é melhor compreendido, soluções projetuais mais eficazes podem ser propostas. Este trabalho mostrou que o Ambiente de Imersão é capaz de oferecer melhores condições para a avaliação da pertinência e adequação das soluções projetuais (pelo melhor entendimento das propostas e de suas repercussões) contribuindo também para a dinamização das reuniões de análise crítica e revisões de projeto, procedimentos fundamentais à coordenação técnica. Consequentemente, pode contribuir também para a racionalização dos processos construtivos ao valorizar e incrementar a fase de concepção e experimentação.

Como discutido neste trabalho, empresas de vários setores já entenderam os benefícios dos Ambientes de Imersão para suas atividades. Essa pesquisa mostrou que os recursos dessa tecnologia podem contribuir também para aumentar a qualidade dos processos da indústria da construção civil, embora essa contribuição esteja sujeita à revisão dos modelos tradicionais de gestão do processo de projeto. É preciso atribuir-lhes a dinâmica inerente ao processo de concepção. Ao permitirem a articulação de conhecimentos entre os membros de uma equipe através do diálogo e do discurso (presencial ou remoto), os Ambientes de Imersão criam o cenário para a consideração e a incorporação do conhecimento tácito de todos os projetistas às propostas conceituais e às soluções técnicas do projeto. Novos modelos de gestão do processo de projeto devem considerar formas mais flexíveis e espontâneas de interação, menos submetidas às relações de controle e poder e mais comprometidas com a finalidade dessas interações (integrar saberes, colaborar).

Essa pesquisa limitou-se em avaliar a capacidade de um sistema de Realidade Virtual Imersiva Exploratória em auxiliar a percepção dos usuários sobre um ambiente virtual. Em estudos futuros nesta área, sugere-se que se avance no desenvolvimento da metodologia para estudos sobre percepção em ambientes de Realidade Virtual e que sejam realizadas investigações sobre a implementação de Ambientes de Imersão como tecnologia de suporte ao longo de todo o processo de projeto de edifícios.

\section{AGRADECIMENTOS}

Ao Departamento de Projetos da Escola de Arquitetura e Design da UFMG. À Professora Dra. Regina Coeli Ruschel da FEC-UNICAMP. Ao CNPq e à CAPES. 


\section{REFERÊNCIAS}

ALVARENGA, L. Representação do conhecimento na perspectiva da ciência da informação em tempo e espaço digitais. Encontros Bibli: Revista Eletrônica de Biblioteconomia e Ciência da Informação. Florianópolis, v. 8, n. 15, p. 18-40, jan/2003. Disponível em:

<https://periodicos.ufsc.br/index.php/eb/article/view/1518-2924.2003v8n15p18>. Acesso em: 14 out. 2013. DOI: http://dx.doi.org/10.5007/1518-2924.2003v8n15p18.

BERTOL, D. Designing Digital Space: An Architect's Guide To Virtual Reality. New York: John Wiley \& Sons, 1997.

BRADY, D.A. The Mind's Eye: Movement and Time in Architecture. In: ACADIA - Anual Conference of the Association for Computer Aided Design in Architecture, 1997, Cincinatti. Proceedings... Cincinatti, out/1997. p. 85-93.

CHANDRASEGARAN, S.K., RAMANIA, K., SRIRAM, R.D., HORVÁTH, I., BERNARD, A., HARIK, R.F., GAO, W. The evolution, challenges, and future of knowledge representation in product design systems. Computer-Aided Design. v. 45, n. 2, p. 204-228, fev/2013.

CRUZ-NEIRA, C. Immersed in science and engineering: Projection technology for high-performance virtual reality environments. In: ICAT - International Conference on Artificial Reality and Telexistence, 1996, Chiba, Japan. Proceedings... Chiba, 1996. p. 77-81.

FAAS, D., BAO, Q., FREY, D.D., YANG, M.C. The influence of immersion and presence in early stage engineering designing and building. Artificial Intelligence for Engineering Design, Analysis and Manufacturing. v. 28, n. 2, p. 139-151, maio/2014.

FLORIO, W. Análise do processo de projeto sob a teoria cognitiva: sete dificuldades no atelier. Arquiteturarevista. São Leopoldo, v. 7, n. 2, p. 161-171, jul/dez 2011.

FRACAROLI, F. Um estudo de percepção em ambiente simulado com realidade virtual exploratória. Dissertação (Mestrado em Engenharia Civil) - Universidade Estadual de Campinas, Faculdade de Engenharia Civil, Arquitetura e Urbanismo, Campinas: 2006. 127 p.

GUIZZARDI, G. Ontological Foundations for Structural Conceptual Models. Tese (Doutorado) Universidade de Twente, Centro de Telemática e Tecnologia da Informação, The Netherlands: 2005.

ISAACS, J.P., GILMOUR, D.J., BLACKWOOD, D.J., FALCONER, R.E. Immersive and Non immersive 3D virtual city: Decision support tool for urban sustainability. ITcon. v. 16, n. especial, p. 151-162, 2011.

KALISPERIS, L., OTTO, G., MURAMOTO, K., GUNDRUM, J., MASTERS, R., ORLAND, B. An Affordable Immersive Environment in Beginning Design Studio Education. In: ACADIA - Annual Conference of the Association for Computer Aided Design in Architecture, 2002, Pomona. Proceedings... Pomona, out/2002. p. 49-56.

LASCARA, C., WHELESS, G., COX, D., PATTERSON, R., LEVY, S., JOHNSON, A.E., LEIGH, J. Tele-Immersive Virtual Environments for Collaborative Knowledge Discovery. In: Advanced Simulation Technologies Conference, 1999, San Diego. Proceedings... San Diego, abr/1999.

MALARD, M.L., RHODES, P.J., ROBERTS, S.E. O Processo de Projeto e o Computador: realidades que interagem virtualmente. Revista de Arquitetura e Urbanismo. Florianópolis, v. 1, n. 1, p. 25-37, 1997.

MALARD, M.L. A lógica do pensamento arquitetônico. EVA - Estúdio Virtual de Arquitetura, 2004. Disponível em: <http://www.arq.ufmg.br/eva/textos.htm>. Acesso em: 29 out. 2013. 
MANZIONE, L., WYSE, M., OWEN, R.L., MELHADO, S.B. Challenges for Implementation of a New Model of Collaborative Design Management: Analyzing the Impact of Human Factor. In: CIB W096 Architectural Management in the Digital Arena, 2011, Wien. Proceedings... Rotterdam, 2011. p. 256-266.

OKAMOTO, J. Percepção Ambiental e Comportamento. São Paulo: IPSIS, 1999.

OLIVEIRA, A.A.S. Utilização da Animação Computacional na Verificação do Programa

Arquitetônico de Necessidades. Dissertação (Mestrado em Engenharia Civil) - Universidade Estadual de Campinas, Faculdade de Engenharia Civil, Campinas: 2003. 154 p.

OWEN, R., AMOR, R., PALMER, M., DICKINSON, J.K., TATUM, C.B., KAZI, A.S., PRINS, M., KIVINIEMI, A., EAST, B. Challenges for Integrated Design and Delivery Solutions. Architectural Engineering and Design Management. v. 6, p. 232-240, nov/2010.

ROMANO, F. Modelo de referência para o gerenciamento do processo de projeto integrado de edificações. Gestão \& Tecnologia de Projetos. São Carlos, v. 1, n. 1, p. 23-46, nov/2006. Disponível em: <http://www.revistas.usp.br/gestaodeprojetos/article/view/50891>. Acesso em: 23 abr. 2013. DOI: http://dx.doi.org/10.4237/gtp.v1i1.7.

RYKEN, M.J., VANCE, J.M. Applying virtual reality techniques to the interactive stress analysis of a tractor lift arm. Finite Elements in Analysis and Design. v. 35, n. 2, p. 141-155, maio/2000.

SCHÖN, D. Educando o profissional reflexivo: um novo design para o ensino e a aprendizagem. Porto Alegre: Artes Médicas Sul, 2000. 256 p.

SILVA, M., NOVAES, C. A coordenação de projetos de edificações: estudos de caso. Gestão \& Tecnologia de Projetos. São Carlos, v. 3, n. 1, p. 44-78, maio/2008. Disponível em:

<http://www.revistas.usp.br/gestaodeprojetos/article/view/50927>. Acesso em: 14 mai. 2013. DOI: http://dx.doi.org/10.4237/gtp.v3i1.28.

STEUER, J. Defining virtual reality: Dimensions determining telepresence. Journal of Communication. v. 42, n. 4, p. 73-93, 1992.

TOBIN, J. Proto-Building: To BIM is to Build. AECbytes. 2008.

WANN, J., MON-WILLIAMS, M. What does virtual reality NEED?: human factors issues in the design of three-dimensional computer environments. International Journal of Human-Computer Studies. v. 44 , n. 6 , p. 829-847, 1996.

WITMER, B., SINGER, M. Measuring presence in virtual environments: A presence questionnaire. Presence. v. 7, n. 3, p. 225-240, 1998. 